\title{
A Study of High-Resolution X-ray Scattering Data Evaluation by the Maximum-Entropy Method
}

\author{
By JÜrgen J. MÜller \\ Max-Delbrück-Center for Molecular Medicine, Robert-Rössle-Strasse 10, D-13125 Berlin, Germany \\ AND STFFn HANSEN* \\ Department of Mathematics and Physics, Royal Veterinary and Agricultural University, \\ Thorvaldsensvej 40, 1871 FRB C, Denmark
}

(Received 26 April 1993; accepted 6 August 1993)

\begin{abstract}
The ability of the maximum-entropy method (in the program MAXENT) to estimate the distancedistribution function from high-resolution X-ray scattering data is studied. It is demonstrated that a key element for the successful application of $M A X E N T$ is the use of a good prior estimate for the distance-distribution function. For simulated as well as experimental data, the effects of different priors, noise levels, smearing and measuring intervals are investigated. For practical applications of $M A X E N T$, various methods for the calculation of priors are treated and a principle for the subsequent choice between the priors is suggested. It is demonstrated that, when the construction of the prior is given sufficient consideration, MAXENT provides a very useful method for estimating the distance distribution from the scattering data.
\end{abstract}

\section{Introduction}

There exist a large number of direct and indirect methods for data treatment and data evaluation of $\mathrm{X}$-ray and neutron scattering experiments (for a review see Glatter, 1982). These procedures have been tested with theoretical and experimental examples for more than a decade. Recently, the maximum-entropy method (MAXENT) has been introduced for the analysis of small-angle scattering data (e.g. Daniell, Potton \& Rainford, 1988a; Hansen \& Pedersen, 1991). MAXENT should provide a powerful tool for the analysis of both small- and wide-angle scattering data. Whereas direct methods suffer from the necessity of explicit extrapolation of the scattering curve to zero and to very large scattering angles, in order to prevent termination errors, the indirect methods working with special functional systems suffer from stability

\footnotetext{
* Work carried out while SH was a visitor at CSIRO, Division of Materials Science and Technology, Australia.

problems when particles of dimensions greater than $10 \mathrm{~nm}$ and resolution better than $0.2 \mathrm{~nm}$ are studied (a task which requires small- as well as wide-angle scattering data). Furthermore, neither of the methods is able to use explicitly any a priori knowledge about the scattering system apart from that of the largest diameter. The maximum-entropy method does not suffer from these limitations. In order to investigate the performance of $M A X E N T$, we have used highly resolved theoretical scattering curves for biological macromolecules. The usual experimental effects encountered, such as quantum noise and data termination at small and large angles, have been simulated and the influences of these as well as of the sampling-point distance on the MAXENT estimate are investigated. The new problems that occur in connection with the use of known structure information, or with the handling of $M A X E N T$ without any explicit structural information, are solved by the introduction of the concept of model priors and intrinsic priors. The structural resolution obtained in the distance-distribution function of a particle with largest diameter of about $8 \mathrm{~nm}$ is circa $0.15 \mathrm{~nm}$. This limit is set by the present parameters chosen for the experimental simulation of the experimental conditions and not by $M A X E N T$ itself.

\section{Theory}

\subsection{Small-angle scattering}

In small-angle scattering, the intensity $I$ is measured as a function of the length of the scattering vector $s=4 \pi(\sin \theta) / \lambda$, where $\lambda$ is the wavelength of the radiation and $\theta$ is half the scattering angle. For scattering from a dilute solution of monodisperse molecules of maximum diameter $D$, the intensity can be written in terms of the distance-distribution function $p(r)$ :

$$
I(s)=\int_{0}^{D} p(r)[\sin (s r) / s r] \mathrm{d} r .
$$


With the distance distribution function $p(r)$ approximated by $\mathbf{p}=\left(p_{1}, \ldots, p_{N}\right)$ and the intensity measured at $\mathbf{s}=\left(s_{1}, \ldots, s_{M}\right)$, the measured intensities are given by

$$
I\left(s_{i}\right)=\sum_{j=1}^{N} A_{i j} p_{j}+e_{i},
$$

where $e_{i}$ is the noise at data point $i$ and the matrix A is given by $A_{i j}=\Delta r \sin \left(s_{i} r_{j}\right) /\left(s_{i} r_{j}\right)$, where $\Delta r=$ $r_{j}-r_{j-1}$. The aim of the indirect Fourier transformation is to restore p, which by virtue of the Fourier transform contains the full information present in the scattering profile.

\subsection{Maximum entropy}

The theoretical background for MAXENT is well established [see e.g. Jaynes (1983) for a review] and just a few results relevant to the present application are mentioned here.

The entropy for a positive additive distribution $\mathbf{p}=\left(p_{1}, \ldots, p_{N}\right)$ with respect to a prior estimate $\mathbf{m}=\left(m_{1}, \ldots, m_{N}\right)$ can be written

$$
S(\mathbf{p}, \mathbf{m})=\sum_{j=1}^{N}-p_{j} \ln \left(p_{j} / m_{j}\right)+p_{j}-m_{j}
$$

[see Skilling (1988) for an axiomatic derivation of this result]. It can be shown that (3) can be obtained from the original entropy measure by assuming each $p_{j}$ to be Poisson distributed with mean $m_{j}$ (Steenstrup, 1985). In cases where the distribution of interest does not have to stay positive, a more appropriate model for the distribution may be obtained by assuming each $p_{j}$ to be Gaussian distributed around a mean $m_{j}$ with width $s_{j}$, and the corresponding expression for the entropy changes to

$$
S(\mathbf{p}, \mathbf{m})=-\sum_{j=1}^{N}\left(m_{j}-p_{j}\right)^{2} / 2 s_{j}^{2}
$$

[for a derivation of how the measure of the information content in a probability density function reduces to (3) and (4) see Kullback (1959)].

It should be noted that in the maximum-entropy method it is the probability distribution for the distribution to be estimated which has to stay non-negative - the distribution itself may take both positive and negative values.

For $s_{j}^{2}=m_{j},(4)$ is the second-order approximation to (3), expressing the fact that the Poisson distribution may be approximated by a Gaussian. For the examples considered in this paper, (3) has been used, except where the prior assumed negative values.

By maximization of the entropy $S$ subject to the constraints from the measured data, the distribution $p$ is found which is closest (as measured by the entropy) to the prior estimate $\mathbf{m}$ while simultaneously fulfilling the constraints from the data. Usually, the constraints from the data are expressed through $\chi^{2}$ given by

$$
\chi^{2}=\sum_{i=1}^{M}\left[I\left(s_{i}\right)-\sum_{j=1}^{N} A_{i j} p_{j}\right]^{2} / \sigma_{i}^{2},
$$

where $\sigma_{i}^{2}$ is the standard deviation for the Gaussian noise at data point $i$. The value for $\chi^{2}$ is required to be equal to a constant $\chi_{a}^{2}$, which represents the degree of misfit considered acceptable. The maximization of the entropy subject to the constraint $\chi^{2}=\chi_{a}^{2}$ is done by standard Lagrange-multiplier techniques through solution of the equations

$$
\nabla\left(S-i \chi^{2}\right)=0,
$$

which - with the equation $\chi^{2}=\chi_{a}^{2}-$ yield $(N+1)$ equations in the $(N+1)$ unknowns $\left(p_{1}, p_{2}, \ldots, p_{N}, \lambda\right)$.

Equation (6) is solved numerically by a successive over/under relaxation algorithm similar to that described by Steenstrup (1985). The algorithm works iteratively by calculation of each new $p_{j}^{\text {ite }+1}$ from the old $p_{j}^{\text {ite }}$, for each $j$, assuming (6) to be an equation in only one unknown $p_{j}$ (using $p_{j}^{\text {ite }+1}$ for $i<j$ and $p_{i}^{\text {ite }}$ for $i>j$ ). At each iteration, $p_{j}^{\text {ite }+1}$ is updated according to $p_{j}^{\text {ite }+1}=(1-\omega) p_{j}^{\text {ite }}+\omega p_{j}$, where $\omega$ is a relaxation factor (usually chosen equal to 0.5 ) and the Lagrange multiplier $\lambda$ is determined by $\hat{\lambda}=|\nabla S| /\left|\nabla \chi^{2}\right|\left(\chi^{2} / \chi_{a}^{2}\right)^{1 / 2}$.

The typical CPU time used for a scattering spectrum of 500 data points and 500 points for the distance-distribution function was about $10 \mathrm{~min}$ on a DEC workstation (equivalent to the speed of a 486 processor). The algorithm used was very stable and there were no problems with the convergence of the algorithm when an appropriate prior was used. If a prior far from the true solution was tried, the program sometimes had problems finding the solution, but no attempt was made to repair this as it did not cause any relevant problems (it simply served as a useful indicator that something was wrong).

For this application of $M A X E N T$, the noise level (and from this the $\chi_{a}^{2}$ ) should be known a priori. If the noise level is not known, the Lagrange multiplier (and the corresponding $\chi_{a}^{2}$ ) can be determined by Bayesian estimation as shown by Gull (1989). For the Bayesian method to give correct results, a 'good' (in the sense of being close to the final estimate) prior is required, but it is also important that the data are free of systematic errors such as, for example, erroneous background subtraction, which may result in spurious structure in the estimated $p(r)$. This last point is not trivial for practical applications of the method to experimental scattering data [see also Bryan (1990) for a further discussion of the Bayesian choice of the Lagrange multiplier]. For the present calculations, the noise level has either been estimated or is known $a$ priori in the case of the simulated examples. 
In $M A X E N T$, the estimation of the errors can be carried out in the conventional way using the usual curvature matrix for the $\chi^{2}$ but including the additional entropy-regularization term as described by Skilling (1989) and Gull (1989). An example of this is given below. However, it has to be noted that systematic contributions to the errors (uncertainty in the validity of the model, background subtraction etc.) are not included in the estimate of the error bars, which should therefore be interpreted conservatively. For the cases considered here, the error estimate is mainly determined by the entropy term. With respect to these problems, MAXENT is similar to other methods for indirect Fourier transformation where the error estimate is conditional on the special functional system chosen for representing the distance-distribution function, the maximum diameter of the scatterer etc.

For the determination of the prior, it has been shown by Skilling (1988) that the 'best' (most likely) prior can be found by maximization of the entropy. This means that any unknown parameters in the prior can be found by maximization of the entropy. For example, if an ellipsoid of revolution is assumed as the prior, the two dimensions can be found as those giving the maximum entropy when the data are fitted to the same $\chi^{2}$ for different choices of dimensions. Or, given the choice between several possible priors, the chosen one should be the one with the maximum-entropy solution when the data are fitted equally closely for the different priors. Examples of both of these situations are given below.

In small-angle scattering, the maximum-entropy method has previously been used for estimation of size distributions (Daniell, Potton \& Rainford 1988a,b; Morrison, Corcoran \& Lewis, 1992) and distancedistribution functions (Hansen \& Pedersen, 1991). To our knowledge, all previous applications of the maximum-entropy method to the determination of size distributions in small-angle scattering have used a uniform prior. Better results can be expected when using a correct prior as indicated by the present calculations (e.g. a Gaussian distribution using a free mean value and free width would be more appropriate as the prior).

\subsection{Direct modified Fourier transformation $(D T)$}

In order to study the performance of $M A X E N T$, scattering curves of biological macromolecules have been calculated from their atomic coordinates stored in the Brookhaven Protein Data Bank (PDB; Bernstein et al., 1977). The improved cube method (Müller, 1983) has been used to calculate the solution scattering, taking into account the solvent-excludedvolume of a molecule. The real-space distance distribution $p(r)$ (Guinier \& Fournet, 1955) has been calculated by a direct Fourier transformation using the theoretical scattered intensity up to $s=30 \mathrm{~nm}^{-1}$. Beyond $30 \mathrm{~nm}^{-1}$, the scattered intensity corresponds to the scattering of the molecule in vacuum and oscillates around the independent-atoms' scattering. The scattered intensity has been damped against the scattering from independent atoms for $s>50 \mathrm{~nm}^{-1}$ with a Hamming window (Müller, Damaschun \& Schrauber, 1990), being zero at $s=100 \mathrm{~nm}^{-1}$. Finally, the independent scattering was subtracted from the scattered intensity of the whole molecule and the difference direct Fourier transformed $(D T)$, corresponding to the inversion of (1):

$$
p(r)=(2 r / \pi) \int_{0}^{\infty} s I(s) \sin (s r) \mathrm{d} s .
$$

By subtraction of the scattering of the independent atoms, only the innermost part of $p(r)$ for $r<0.4 \mathrm{~nm}$ is influenced, because the largest van der Waals radii used are half this size. The slit-length smearing was carried out by numerical integration

$$
J(t)=2 \int_{0}^{t_{\max }} P(t) I\left[\left(s^{2}+t^{2}\right)^{1 / 2}\right] \mathrm{d} t
$$

with the primary-beam-length profile

$$
P(t)=c \pi^{-1 / 2} \exp \left(-c^{2} t^{2}\right) .
$$

$P\left(t_{\max }\right) / P(0)=10^{-5}$ and $c$ was chosen to be $0.6863 \mathrm{~nm}$. The addition of random noise, the slit-length smearing and the nonlinear curve fitting to rotational ellipsoids by Marquardt's (1963) procedure were carried out using the computer program package $S A X S-P C$ (Müller, 1992).

\section{Results}

The first test example is the theoretical scattering curve of phenylalanine-specific transfer ribonucleic acid (tRNA ${ }^{\text {Phe }}$ ) using the coordinate set 4TRA deposited in the PDB by Westhof, Dumas \& Moras (1988). Random noise has been added with a constant relative value of 10,5 and $1 \%$ of the intensity, respectively. The full curves are shown in Fig. 1 for pin-hole collimation as well as for slit geometry. The main point before the investigation of the influences of the experimental restrictions on the results of the desmearing and transformation by $M A X E N T$ is the determination of a correct prior. Without a priori knowledge of the atomic model, several priors are possible, at first priors are derived from low-resolution models like homogeneous spheres, ellipsoids or aggregates of finite elements. By Marquardt's curve-fitting procedure for $0 \leq s \leq 1.2 \mathrm{~nm}^{-1}$, an ellipsoid of revolution with half-axes $A=4.81$ and $B=C=1.63 \mathrm{~nm}$ was found as a first crude model. From this ellipsoid, a structurally more realistic 
triaxial ellipsoid, the inertia-equivalent ellipsoid $(A=4.6, B=2.75, C=1.38 \mathrm{~nm})$, can be estimated from the scattering curve by calibration (Müller \& Schrauber, 1992). Both scattering curves are shown in Fig. 2. Ellipsoids do not fit the complicated tRNA structure and consequently their scattering curves are bad approximations. The corresponding distancedistribution functions of these models $p(r)$ are used as priors and are shown in Fig. 3. Per definitionem, we call all priors derived from models model priors. Both model priors are far from the high resolution $p(r)$ of

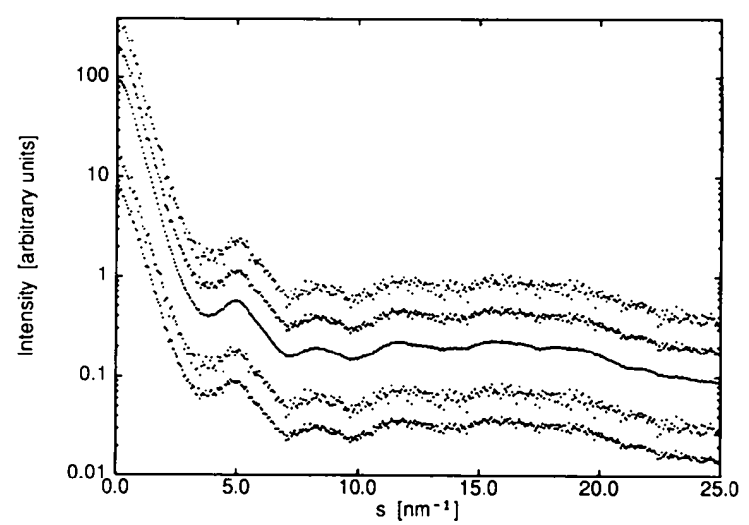

Fig. 1. Theoretical scattering curves of transfer ribonucleic acid tRNA ${ }^{\text {Phc }}$ calculated from atomic coordinates of the PDB entry 4TRA. The upper three curves are for pinhole collimation and 10,5 and $1 \%$ relative random noise. The lower two curves are slit-length smeared with a Gaussian profile and 10 and $5 \%$ random noise. The curves are shifted vertically by arbitrary values. the tRNA molecule. The scattering of the structurally more adequate inertia-equivalent ellipsoid differs more at smaller angles from the tRNA curve than the scattering of the equivalent ellipsoid of revolution. The corresponding result can be seen for the priors. The $p(r)$ of the inertia-equivalent ellipsoid shows greater large-distance differences from the highresolution function than $p(r)$ of the rotational ellipsoid. Fig. 4 shows the $M A X E N T$ results for both priors using the $5 \%$-noise data. The priors stamp the results. The global differences between the true $p(r)$ and the priors cannot be removed by the MAXENT procedure. The fine structure of $p(r)$ is also misleading for both priors.

The results for the homogeneous ellipsoids are unsatisfactory and must be rejected. The systematic errors from simple models, usually known a priori or estimable directly from the scattering curve, may propagate into the $M A X E N T$ result.

The second possibility to derive a convenient prior without using a (possibly) wrong model is to use the scattering curve itself with a lower resolution than obtained experimentally. We use the original data (Fig. 1) and extrapolate the curve corresponding to the Porod law from $s=2.88 \mathrm{~nm}^{-1}$ to infinity or alternatively apply an indirect method for data handling, e.g. the program ITP developed by Glatter (1977). The $p(r)$ function derived from the measured data by such a procedure is called an intrinsic prior. This prior fits the high-resolution $p(r)$ very well in the overall curve shape and lacks only the fine structure (Fig. 3). The result for $M A X E N T$, when this intrinsic prior is used, is shown in Fig. 5. The fine structure is

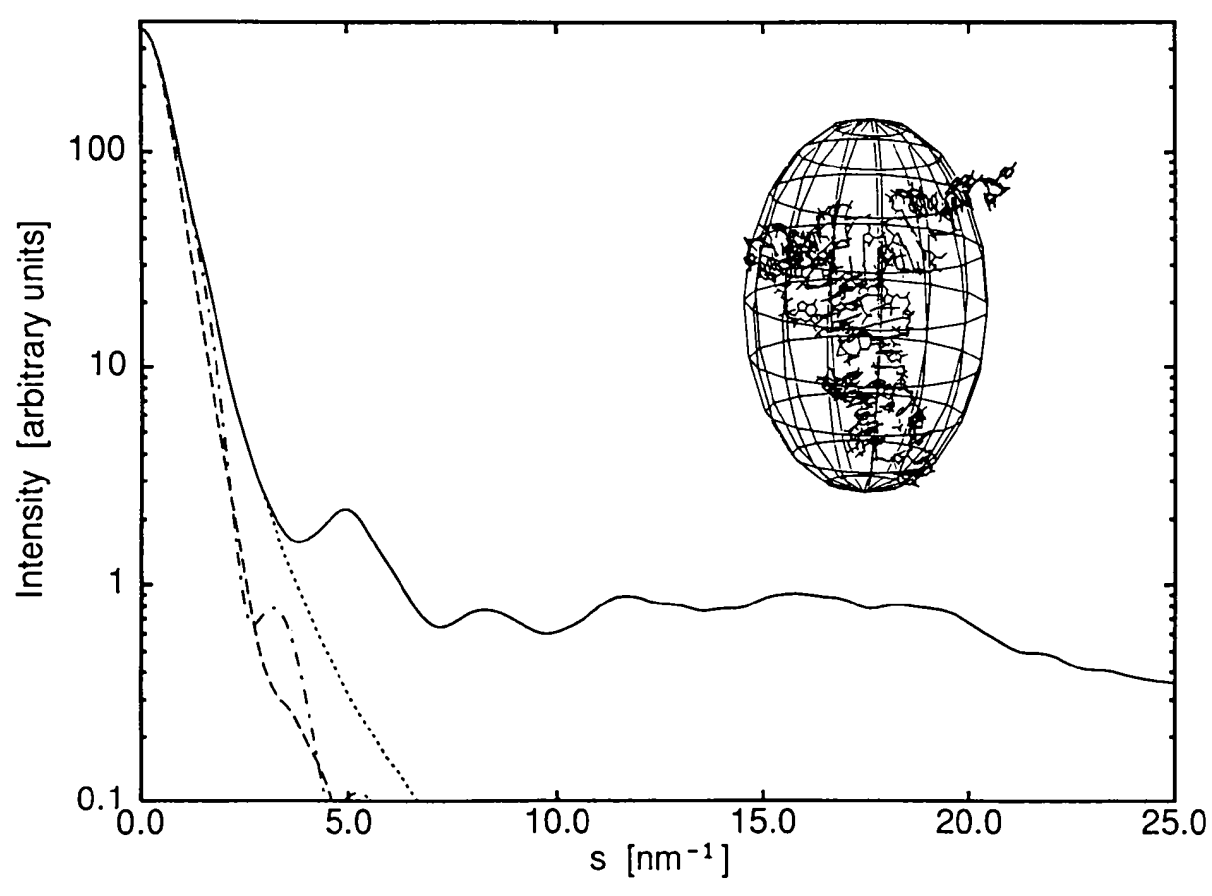

Fig. 2. Scattering curves of the tRNA and of different priors. Full line: tRNA, crystal structure (4TRA model in insert). Dasheddotted line: ellipsoid of revolution $(A=4.81, B=C=1.63 \mathrm{~nm})$. Long dashes: inertia-equivalent ellipsoid $(A=4.6, B=2.75, C=$ $1.38 \mathrm{~nm})$. Short dashes: lowresolution scattering curve, $s^{-4}$ law from $s=2.88 \mathrm{~nm}^{-1}$. 
restored without spurious oscillations and the overall agreement of the high-resolution $p(r)$ with the $M A X E N T$ result is adequate for further discussions.

For investigation of the sensitivity of $M A X E N T$ against noise, angular step width and termination errors, we use the intrinsic low-resolution prior.

Fig. 6 shows $M A X E N T$ results for 1,5 and $10 \%$ constant relative noise added to the scattering curves. The fine structure is very well restored for the example using the lowest noise level. As the noise level is increased, the fine structure is smeared out in a well behaved manner as the details disappear and the estimate is pulled closer to the prior by the entropy term in (6). The increased noise level allows the

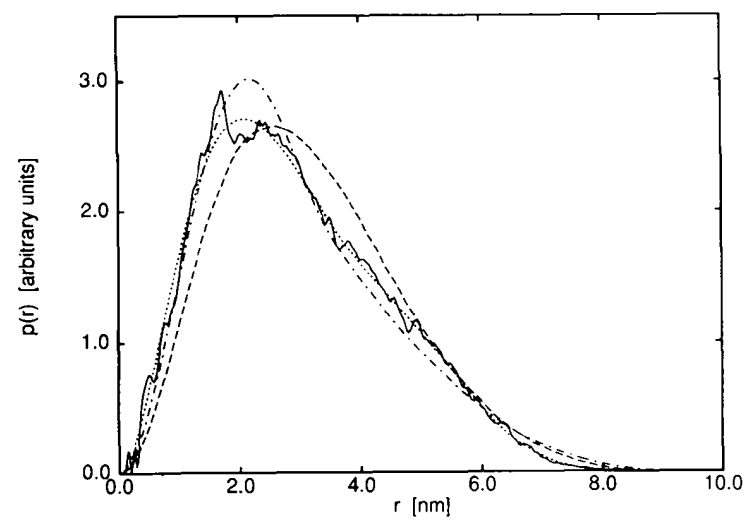

Fig. 3. High-resolution distance distribution of tRNA and of different priors. Full line: $p(r)$ of tRNA (crystal structure, 4TRA). Dashed-dotted line: $p(r)$ of the ellipsoid of revolution $(A=4.81$, $B=C=1.63 \mathrm{~nm})$. Long dashes: $p(r)$ of the inertia-equivalent ellipsoid $(A=4.6, B=2.75, C=1.38 \mathrm{~nm})$. Short dashes: intrinsic low-resolution prior.

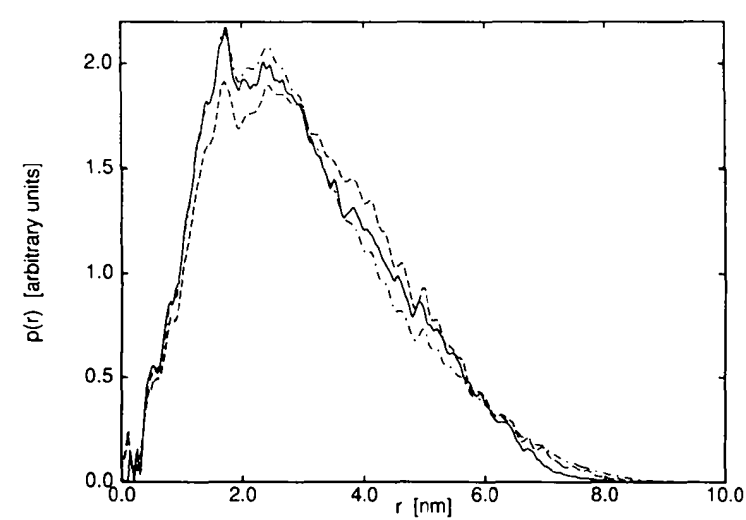

Fig. 4. MAXENT result for tRNA using model priors. Full line: $p(r)$ of tRNA (crystal structure, 4TRA). Dashed-dotted line: $M A X E N T$ result using the ellipsoid of revolution as prior. Long dashes: $M A X E N T$ result using the inertia-equivalent ellipsoid as prior.

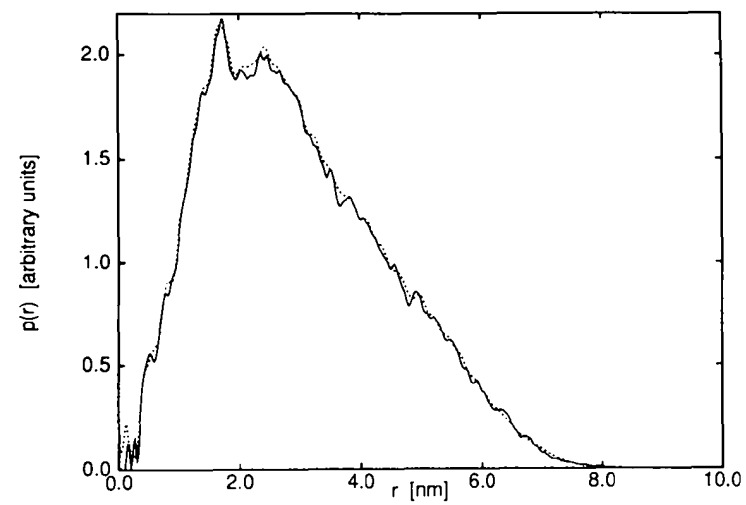

Fig. 5. $M A X E N T$ result for $\mathrm{tRNA}$ using an intrinsic prior. Full line: $p(r)$ of tRNA (crystal structure, 4TRA). Short dashes: $M A X E N T$ result using the intrinsic low-resolution prior (see Fig. 3).

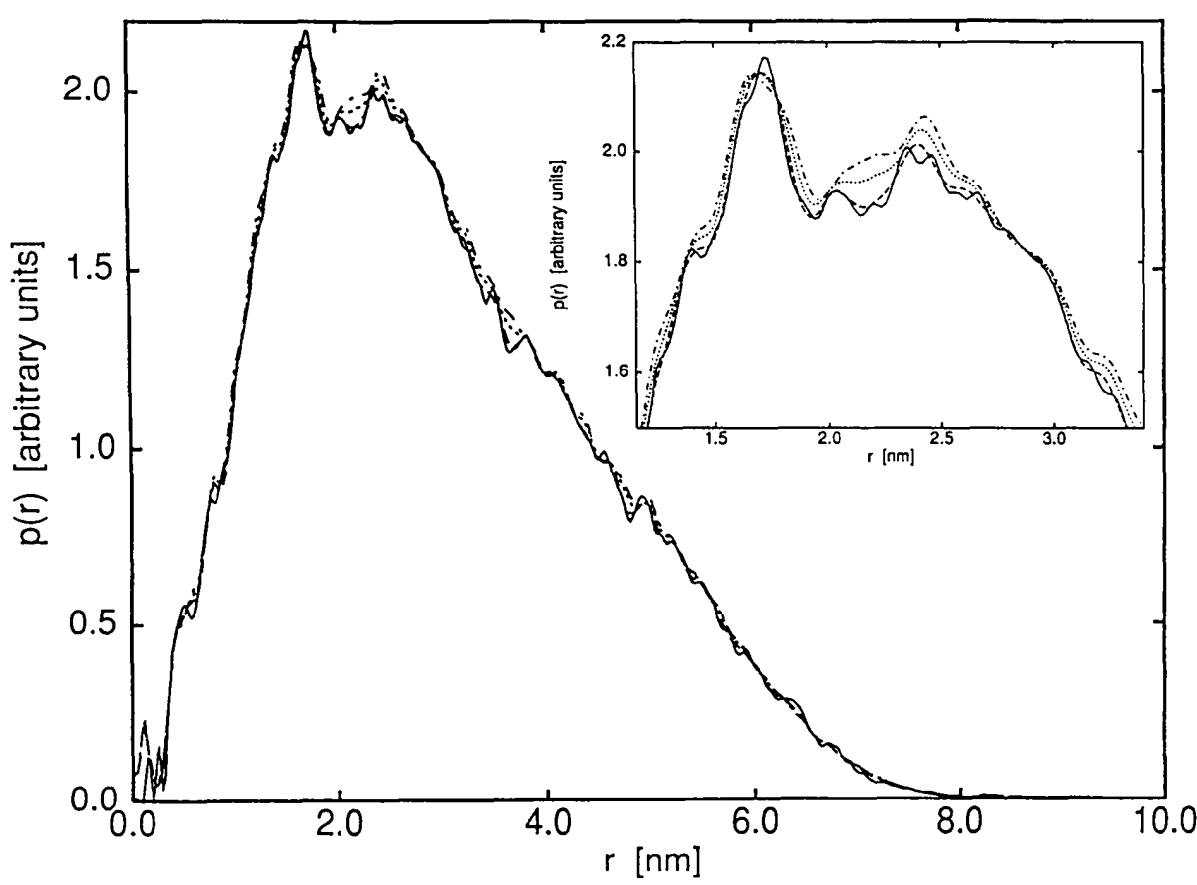

Fig. 6. $M A X E N T$ result for $t$ RNA using the low-resolution intrinsic prior and scattering curves with different constant relative noise. Full line: $p(r)$ of $t$ RNA (crystal structure, 4TRA). Long dashes: $1 \%$ noise. Short dashes: $5 \%$ noise. Dashed-dotted line: $10 \%$ noise. 
entropy term in (6) to be more dominant as the constraints from the data are weaker. In the limit of no constraint from the data, the prior will be refound as the solution to (6). This controlled behaviour of the solution is one of the virtues of $M A X E N T$. In the presence of an incorrect prior, artifacts may appear, but when a good prior has been used $M A X E N T$ is not likely to cause spurious structure in the estimated $p(r)$. For a constant absolute noise of $0.027 \%$ of $I(0)$ over the whole curve (Fig. 7 , insert), the fine structure is somewhat smeared out but essentially the same as in the high-resolution $p(r)$ (Fig. 7).

In the calculations discussed above, the scattering curve of tRNA was given for $s=0-23 \mathrm{~nm}^{-1}$ at intervals of $\Delta s=0.06 \mathrm{~nm}^{-1}$, which means at about $1 / 6$ of the sampling-point distance as in most experiments. Fig. 8 shows that the main features are

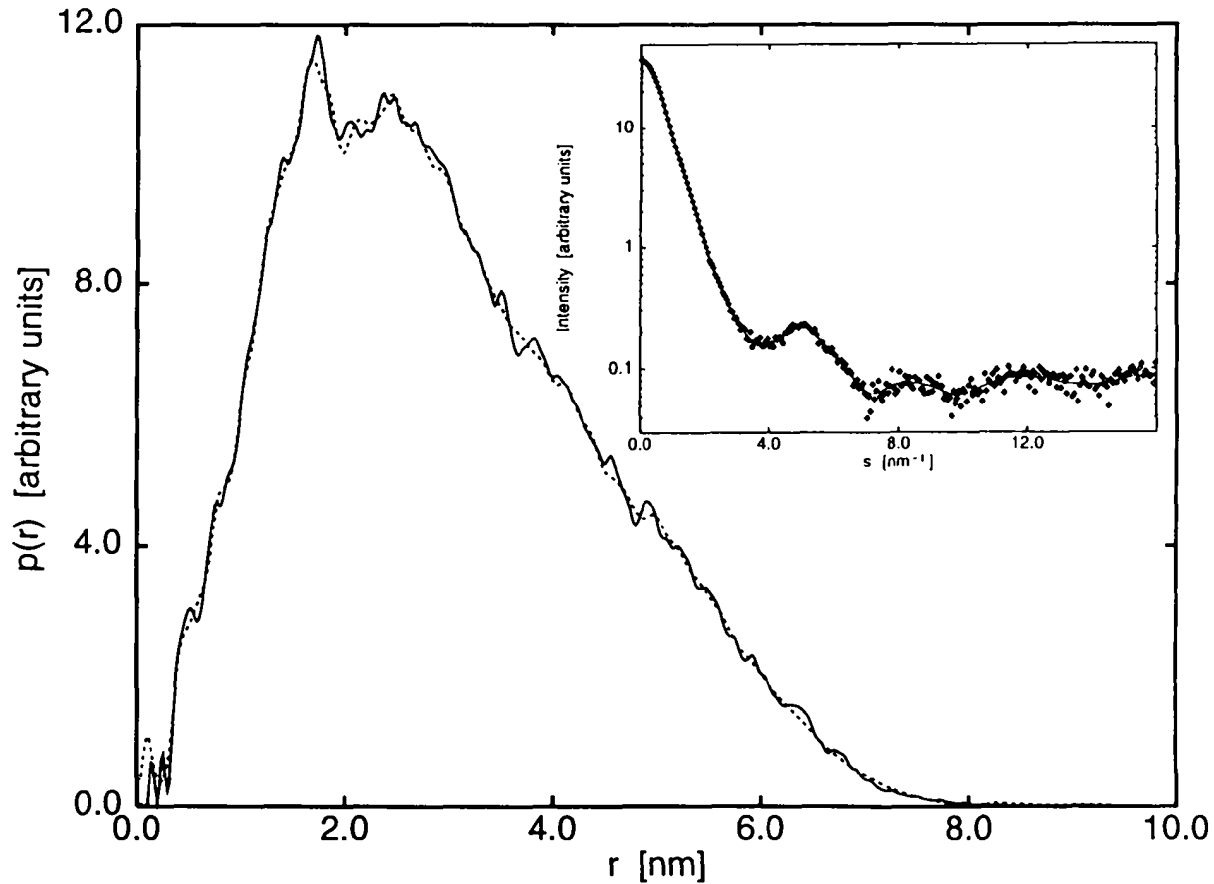

Fig. 7. $M A X E N T$ result for tRNA using the low-resolution intrinsic prior and a scattering curve with $0.00027 I(0)$ absolute random noise. Full line: $p(r)$ of tRNA (crystal structure, 4TRA). Dashed line: $M A X E N T$ estimate. Insert: scattered intensity of tRNA (crystal structure, 4TRA) with an absolute random noise $0.00027 I(0)$. Full line: $M A X E N T$ fit.

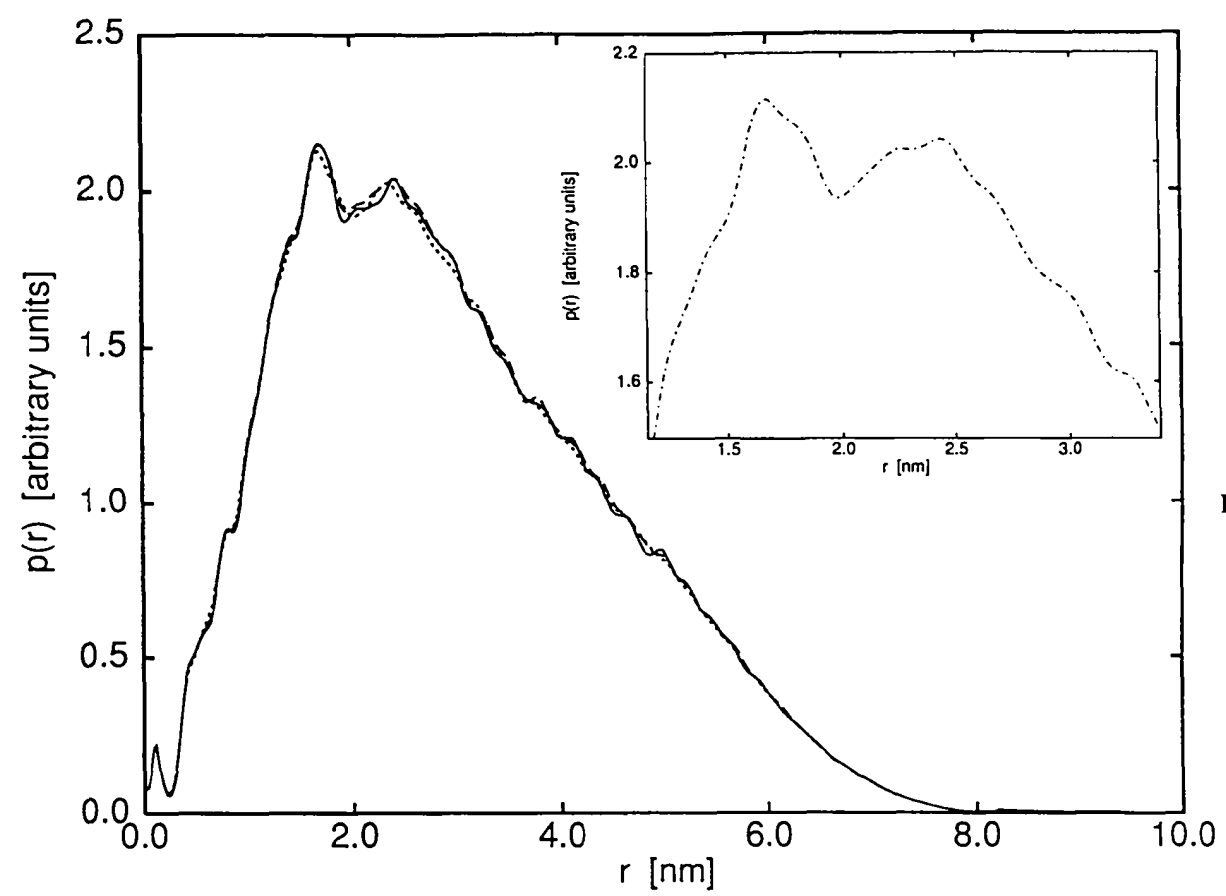

Fig. 8. $M A X E N T$ result for tRNA using the low-resolution intrinsic prior and scattering curves with different angular step width $(5 \%$ constant relative noise). Full line: step width $\Delta s=0.06 \mathrm{~nm}^{-1}$. Long dashes: step width $\Delta s=$ $0.12 \mathrm{~nm}^{-1}$. Short dashes: step width $\Delta s-0.36 \mathrm{~nm}^{-1}$. Insert: maximum region of $p(r)$ for scattering data with $10 \%$ constant relative noise and step width $\Delta s=0.12 \mathrm{~nm}^{-1}$. 
preserved for the step widths used, $\Delta s=0.06,0.12$ and $0.36 \mathrm{~nm}^{-1}$, respectively, for a constant relative noise of $5 \%$. The fine structure in $p(r)$, especially in the region $1.8-2.5 \mathrm{~nm}$, is reproduced best for the scattering data with the highest information content (step width $0.06 \mathrm{~nm}^{-1}$ ). With $10 \%$ relative noise and step width $\Delta s=0.12 \mathrm{~nm}^{-1}$, the information about the fine structure is lost, as shown in the insert.

In Fig. 9, the simulated intensity (again for 5\% noise) has been truncated, now with $s_{\min }=1.8 \mathrm{~nm}^{-1}$ as the first point. This corresponds to five times the sampling-point distance $\pi / L$. However, as the prior has been estimated using the original data up to

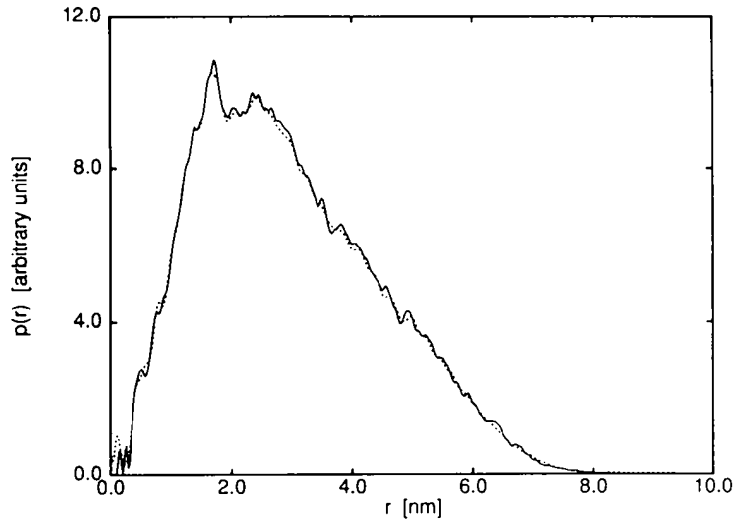

Fig. 9. $M A X E N T$ result for tRNA using the low-resolution intrinsic prior and a scattering curve with $5 \%$ constant relative noise using $s_{\min }=1.8 \mathrm{~nm}^{-1}$. Full line: $p(r)$ of tRNA (crystal structure, 4TRA). Short dashes: MAXENT result. $s=2.88 \mathrm{~nm}^{-1}$, it contains the information corresponding to the missing data points. Therefore, the $M A X E N T$ estimate of $p(r)$ is again satisfactory.

\subsection{Curve extrapolation}

The behaviour of the $M A X E N T$ results has been investigated for scattering curves terminated at different angles at the large-angle end. To simulate the accessible angular region of a diffractometer, the scattering curves were truncated in minima as well as in maxima. The scattering curves are perfectly fitted by $M A X E N T$ up to the corresponding termination angle (Fig. 10). The extrapolated part of the scattering curve oscillates around an $s^{-4}$ tail, containing some spurious maxima, the stronger, the lower the resolution - simulating a homogeneously filled hard-body model with a sharp electron-density boundary. The termination angle $s_{\max }$ is not important to the convergence or the quality of the MAXENT results [the $p(r)$ functions in Fig. 11 correspond to the resolution $\left.\pi / s_{\max }\right]$.

\subsection{Slit-length desmearing}

The smearing and desmearing effects will be investigated in more detail in a later study. Here, we make only a brief remarks concerning the slit-length desmearing of a tRNA curve. The scattering curve derived from the atomic coordinate set 4TRA has been smeared by a profile $P(t)$ corresponding to (9) with a simulation program incorporated in $S A X S-P C$. $5 \%$ random noise has been added. The curve is shown

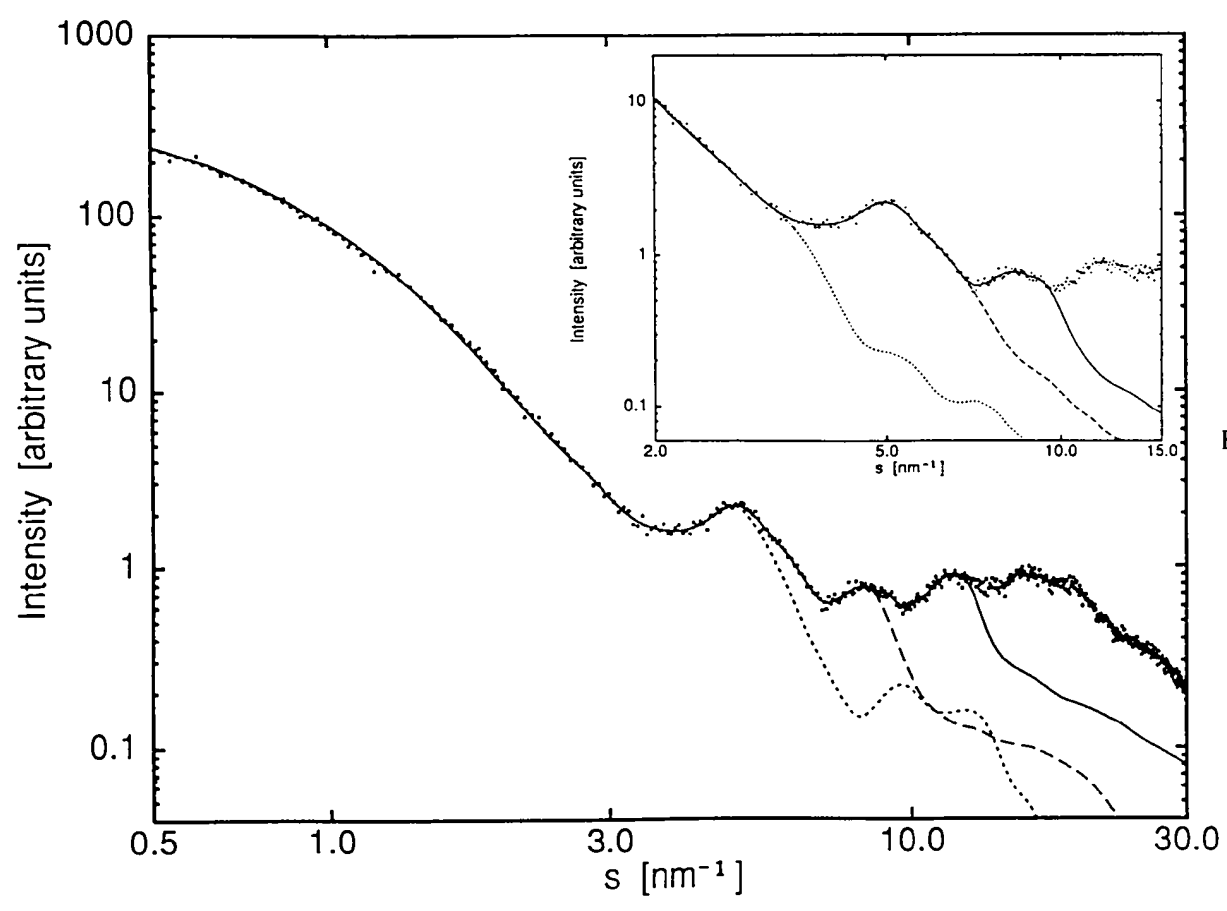

Fig. 10. $M A X E N T$ result for $\mathrm{RNA}$ (crystal structure, 4TRA) using the low-resolution intrinsic prior and scattering curves with $5 \%$ constant relative noise terminated at different larger angles. Markers show the data for $T$ RNA. Full line: $M A X E N T$ extrapolation for a termination at $s_{\max }=$ $12.5 \mathrm{~nm}^{-1}$. Long dashes: $s_{\max }=$ $8.5 \mathrm{~nm}^{-1}$. Short dashes: $s_{\max }=$ $5.0 \mathrm{~nm}^{1}$. Full line in insert: $s_{\max }=9.7 \mathrm{~nm}^{-1}$. Long dashes in insert: $s_{\max }=6.8 \mathrm{~nm}^{-1}$. Short dashes in insert: $s_{\max }=3.4 \mathrm{~nm}$ 
in Fig. 1. It was used as input data for the indirect transformation method ITP (Glatter, 1977) up to $s=3.4 \mathrm{~nm}^{-1}$. The $p(r)$ obtained was then used as a low-resolution prior. Fig. 12 shows the results for $M A X E N T$. There is a complete agreement for the $p(r)$ functions based on pin-hole-collimated and slitlength-smeared data.

\subsection{Priors for globular particles}

For the branched molecule tRNA, the simple model prior in the form of an ellipsoid was not adequate but the intrinsic low-resolution prior

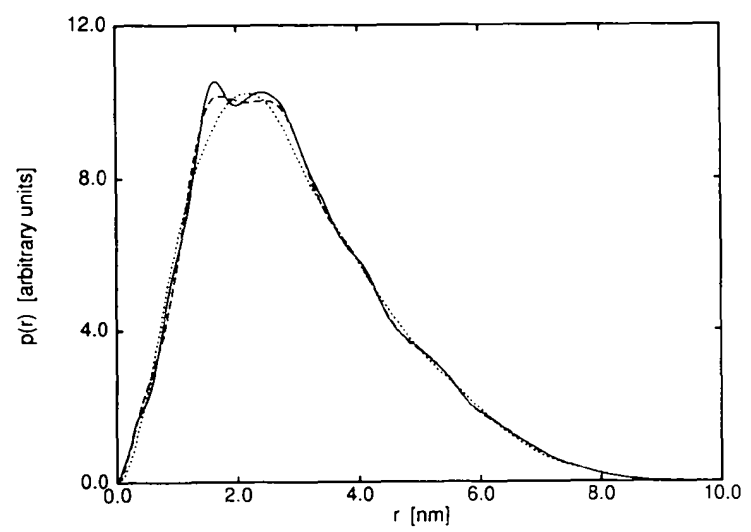

Fig. 11. MAXENT result for tRNA (crystal structure, 4TRA) using the low-resolution intrinsic prior and scattering curves with $5 \%$ constant relative noise and reduced resolution. Full line: termination at $s_{\max }=9.7 \mathrm{~nm}^{-1}$. Long dashes: $s_{\max }=6.8 \mathrm{~nm}^{-1}$. Short dashes: $s_{\max }=3.4 \mathrm{~nm}^{-1}$. worked very well. Now, we consider an example where the reverse is true. Fig. 13 shows the scattering curve of the globular protein cytochrome c calculated from the atomic coordinates of the PDB entry 1CYC (Tanaka, Yamane, Tsukihara, Ashida \& Kakudo, 1975). If the rotational ellipsoid with the half-axes $A=1.9, B=C=1.5 \mathrm{~nm}$ is chosen as model prior, we get a $M A X E N T$ result showing all main fine-structure features apart from the smaller ripples for $r \leq 3 \mathrm{~nm}$ (Fig. 14). There is no large-scale discrepancy between the high-resolution $p(r)$ and the $M A X E N T$ result, because the model prior is adequate for the molecule.

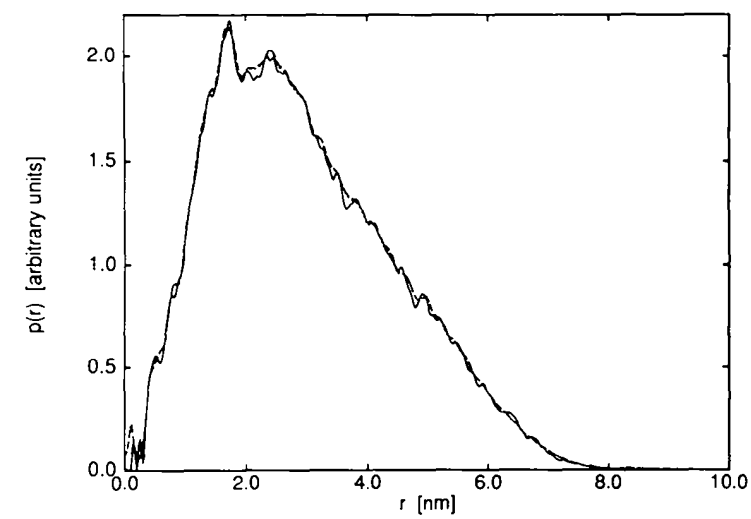

Fig. 12. MAXENT result for tRNA using low-resolution intrinsic prior and pin-hole collimated and slit-length-smeared scattering curves with $5 \%$ constant relative noise. Full line: $p(r)$ of tRNA (crystal structure, 4TRA). Long dashes: MAXENT result for pin-hole-collimated scattering curve. Short dashes: MAXENT result for slit-length-smeared scattering curve.

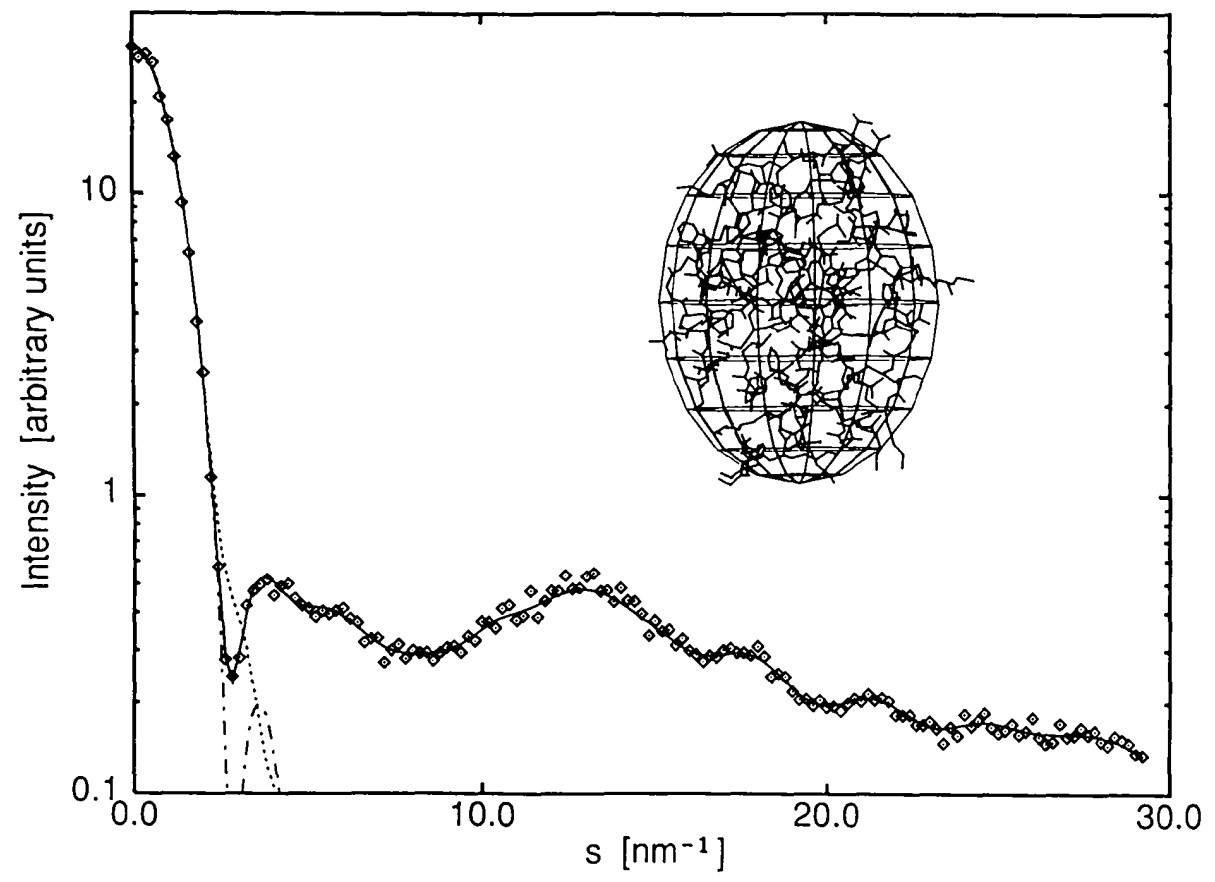

Fig. 13. Scattering curve of cytochrome c calculated from atomic coordinates of the PDB entry 1CYC. $5 \%$ constant relative noise has been added. Full lines: MAXENT fit. Short dashes: Fourier transform of low-resolution prior. Dashed-dotted line: Fourier transform of rotational ellipsoid. Insert: atomic resolved structure of $1 \mathrm{CYC}$ and rotational ellipsoid with half-axes $A=1.9$, $B=C=1.5 \mathrm{~nm}$. 
It has been determined by maximization of the entropy as described above and fits the crystal structure of 1CYC very well (see insert in Fig. 13).

Contrary to this, the low-resolution intrinsic prior fails when an incorrect truncation of the scattering curve is chosen. Fig. 13 shows the $s^{-4}$ extrapolation starting at $s=2.2 \mathrm{~nm}^{-1}$. The resulting $p(r)$ function shows truncation errors. For large distances, the prior has negative values. Using $M A X E N T$ and (3), the $p(r)$ can be constrained to stay positive but as noted previously this is not mandatory. If the positivity constraint is omitted, the MAXENT estimate will follow the negative prior (Fig. 15). However, the main features of $p(r)$ are restored again within the region $0.4 \leq r \leq 2.8 \mathrm{~nm}$.

If there exists a priori an adequate low-resolution model or if there exists a high probability for a compact isometric structure, hard bodies may be used as suitable priors. In general, as demonstrated by all the examples given here, the MAXENT estimate will follow the overall structure of the prior, which therefore should not contain any artifacts if $M A X E N T$ is to perform satisfactorily.

\subsection{Low-resolution intrinsic prior compared with high-resolution model prior}

To study the role of the prior and the method for calculation of an intrinsic prior, we simulated an experiment with $t \mathrm{RNA}^{\mathrm{Phe}}$. It is known from theoretical potential and energy calculations by Harvey \& McCammon (1981) that tRNA can undergo largescale global conformational changes with low energetic costs. To model a structural change, the aminoacyl stem of the molecule has been rotated against the anticodon stem around the axis connecting the P8 and P49 atoms. The rotation angle has been chosen to be 32 , to achieve a clear effect in the

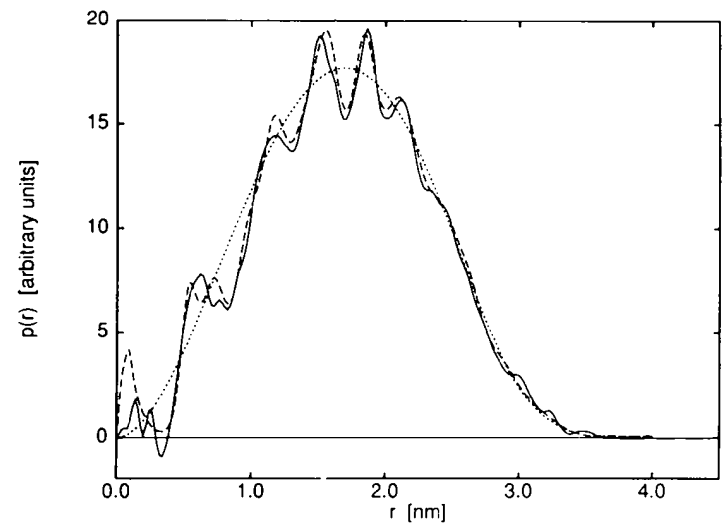

Fig. 14. MAXENT result for cytochrome c (1CYC) using the model prior ellipsoid of revolution and a scattering curve with $5 \%$ constant relative noise. Full line: $p(r)$ of $1 \mathrm{CYC}$. Short dashes: model prior ellipsoid of revolution. Long dashes: $M A X E N T$ result. scattering curve (Fig. 16) and distance-distribution function $p(r)$ (Fig. 17). 5\% constant relative random noise was added to the scattering curve of the modified $t R N A$.

The idea was to use the highly resolved $p(r)$, which was calculated from the crystal structure (coordinate set 4TRA) by the modified direct Fourier transformation described above, as a prior for the modified tRNA structure. Fig. 18 shows the $M A X E N T$ result. The fine structure of the $M A X E N T$ result is very similar to that expected but the global differences visible in Fig. 17 are manifested by the wrong model prior. Also, a medium-resolution model prior, calculated from the scattering curve of the tRNA (coordinate set 4TRA), after extrapolation $\left(s^{-4}\right.$ tail starting at $\left.s=6.9 \mathrm{~nm}^{-1}\right)$ by the direct Fourier transformation has to be rejected (Fig. 19). The large-scale differences remain the same and additionally the fine structure is somewhat flattened out because of the reduced resolution in the prior. Therefore, as the high-resolution model prior has failed, we have to choose a lower-resolution intrinsic prior. Fig. 20 shows the result for the low-resolution prior that was calculated from the scattering curve within $s=0-2.88 \mathrm{~nm}^{-1}$ by a direct Fourier transformation. The agreement of the $M A X E N T$ result and the expected $p(r)$ is satisfactory.

The error bars, shown in the insert, have been plotted at intervals corresponding to the resolution of the data and indicate the concentration of distributions at the MAXENT solution. The regions where the data contained sufficient information to pull the estimate away from the prior are evident in the figure by comparison with the size of the error bars. However, again it should be noted that the error bars are conditional on the prior estimate.

The same result (not shown here) was obtained when an intrinsic prior of medium resolution $\left(s_{\max }=6.9 \mathrm{~nm}^{-1}\right)$ was used.

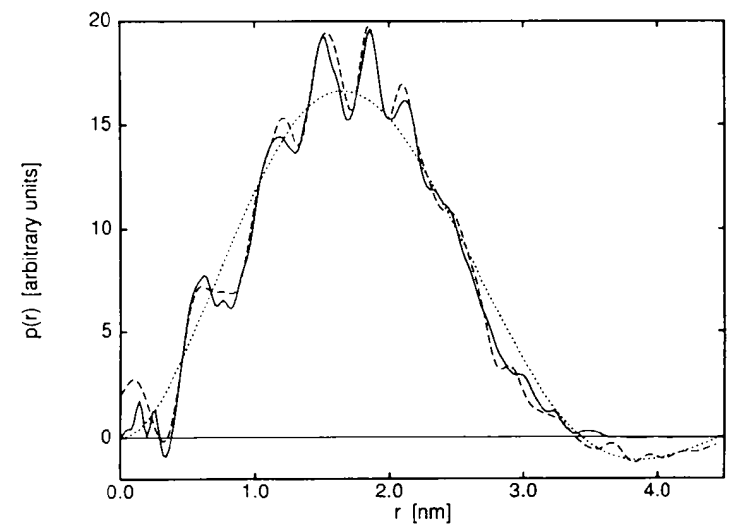

Fig. 15. MAXENT result for cytochrome c (1CYC) using a low-resolution intrinsic prior and a scattering curve with $5 \%$ constant relative noise. Full line: $p(r)$ of $1 \mathrm{CYC}$. Short dashes: low-resolution intrinsic prior. Long dashes: $M A X E N T$ result. 
Next, the method for calculation of the prior was changed to study the influence of the extrapolation of the scattering curve to larger angles. The direct method fits a power-law constant in $a / s^{4}$ by a least-squares procedure in a local region of the scattering curve. The indirect methods extrapolate the scattering curve to large angles by the information content in the special functional system fitted to the full known curve. But by ITP it is not possible to calculate the highly resolved $p(r)$ from the curve with $5 \%$ random noise in the angular region $0 \leq s \leq$
$27 \mathrm{~nm}^{-1}$ for a molecule with a maximum diameter of $8 \mathrm{~nm}$. Stability problems result in strong oscillations in $p(r)$ (using 40 splines). But low-resolution and medium-resolution priors for use in $M A X E N T$ can be determined with Glatter's program ITP. At first, a low-resolution model prior derived from the 4TRA scattering curve was calculated by ITP. The result (Fig. 21) is the same as for a medium-resolution model prior (not shown here) and corresponds to the result for the prior calculated by the direct method (Fig. 19). The global discrepancies are visible again. The best

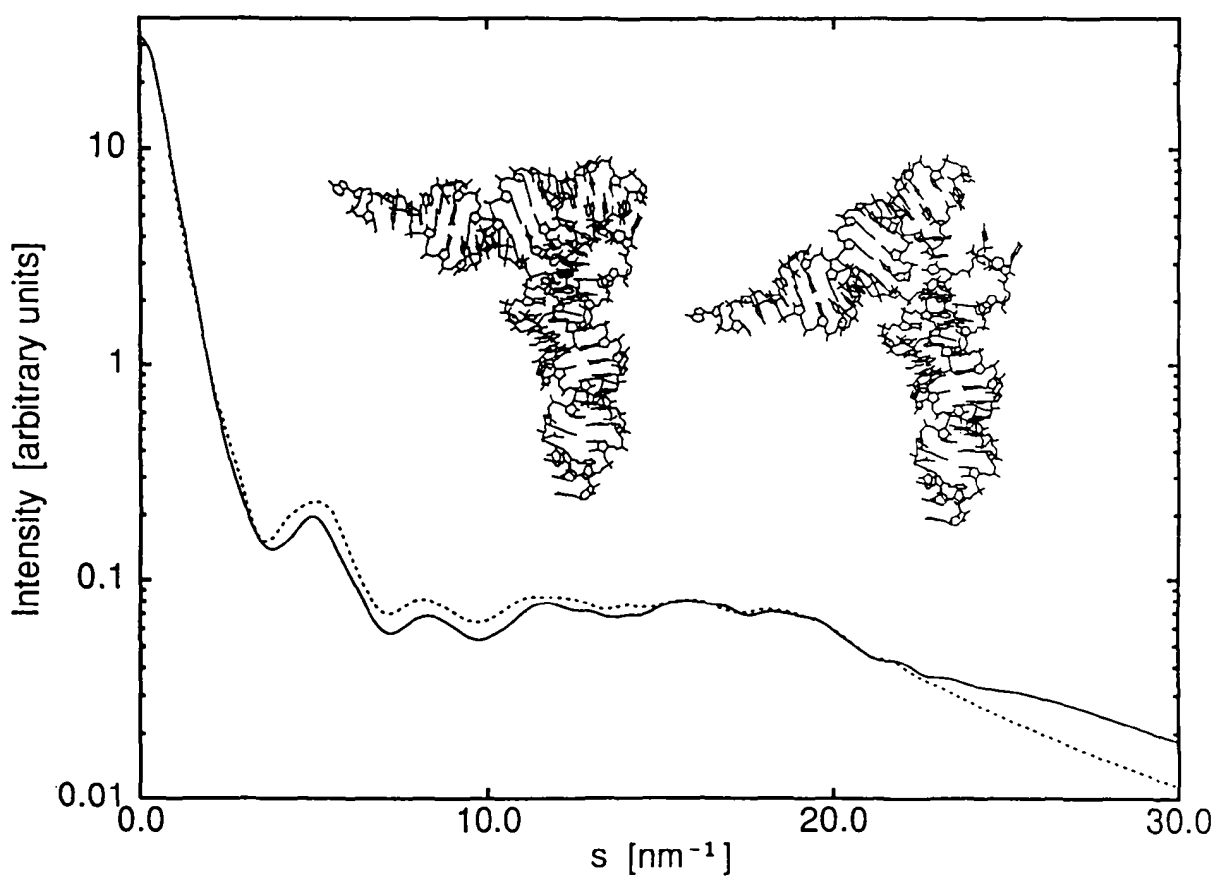

Fig. 16. Scattering curves for tRNA (crystal structure, 4TRA) and modified tRNA. Full line: tRNA scattering curve calculated from atomic coordinates of the PDB entry 4TRA. Dashes: scattering curve of the modified molecule. Insert: left 4TRA, right aminoacyl arm rotated around the P8-P49 axis by $32^{\circ}$.

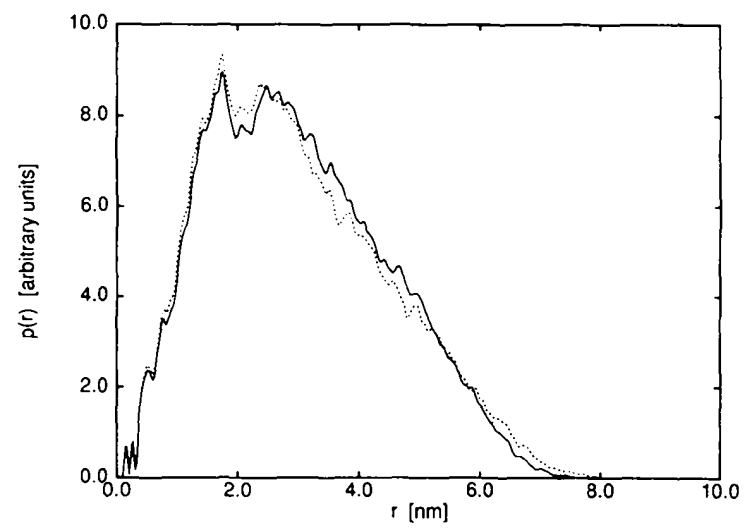

Fig. 17. High-resolution distance-distribution functions for both the tRNA models shown in Fig. 16, calculated by a modified direct Fourier transformation. Full line: $p(r)$ for the modified tRNA structure. Short dashes: $p(r)$ for tRNA (crystal structure, 4TRA).

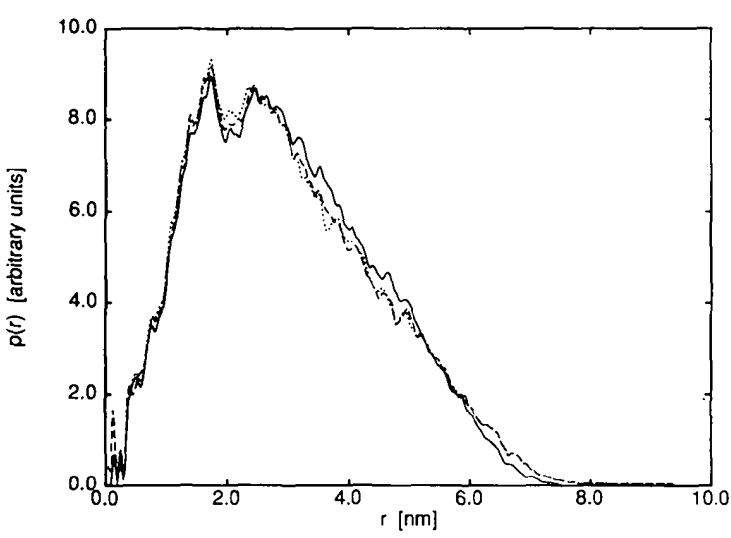

Fig. 18. MAXENT result for a modified $t$ RNA structure using the distance-distribution function of the TRNA with crystal structure (4TRA) as high-resolution model prior. Full line: $p(r)$ of the modified tRNA structure, calculated by a modified direct Fourier transformation. Short dashes: high-resolution model prior (crystal structure, 4TRA), calculated by a modified direct Fourier transformation. Long dashes: $M A X E N T$ result. 
result for the modified structure was obtained when the low-resolution intrinsic prior, calculated by ITP from the scattering curve of the modified structure $\left(0 \leq s \leq 2.88 \mathrm{~nm}^{-1}\right)$, was used. The global as well as the in-detail agreement are visible in Fig. 22. Especially for distances larger than $5.5 \mathrm{~nm}$, the $M A X E N T$ result is an improvement compared with the result in Fig. 20. Because the result is identical with the $p(r)$ calculated for a medium-resolution intrinsic prior $\left(0 \leq s \leq 6.9 \mathrm{~nm}^{-1}\right)$ (not shown), the fading out of some fine structure (e.g. the small peak

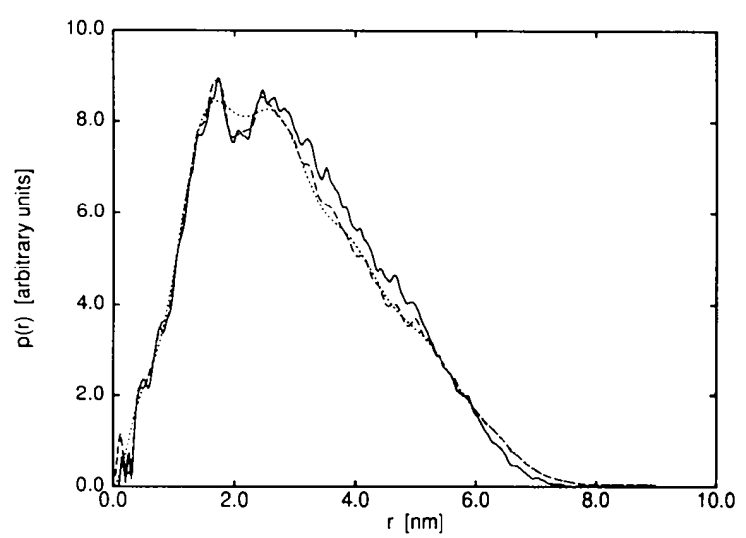

Fig. 19. MAXENT result for a modified tRNA structure using the medium-resolution distance distribution of the tRNA with crystal structure (4TRA) as model prior. Full line: $p(r)$ of the modified tRNA structure. Short dashes: medium-resolution prior, calculated by direct Fourier transformation. Long dashes: $M A X E N T$ result. at $2 \mathrm{~nm}$ ) is caused by the random noise acting in an identical manner for both priors. If nothing is known about the correct $p(r)$ function, the entropy may be taken as a relative measure for the adequacy of the prior. Table 1 contains the numerical values of the entropy, which confirm the ability of MAXENT to 'rank' the performance of the different priors by their relative value for the entropy.

\subsection{Experimental $t R N A^{\text {Phe }}$}

The experimental $\mathrm{TRNA}^{\text {Phe }}$ scattering curve was measured in the angular region $0.0782 \leq s \leq$ $15.55 \mathrm{~nm}^{-1}$ (Müller, Damaschun, Wilhelm, Welfle \& Pilz, 1982). Fig. 23 shows the point-collimated experimental curve in comparison with the theoretical scattering curve calculated from the crystal structure (atomic coordinate set 4TRA). In Fig. 23 (insert), the $p(r)$ functions that have been used as priors for $M A X E N T$ are depicted. Fig. 24 shows the results in comparison with the $p(r)$ function derived from the crystal structure. The $M A X E N T$ result for the ellipsoidal model prior determined by curve fitting for $s \leq 1.2 \mathrm{~nm}^{-1}$ should be rejected.

A slight shift (about $0.07-0.08 \mathrm{~nm}$ ) of the peak at $1.75 \mathrm{~nm}$ appears in the calculated distance-distribution function. This shift is also present if the better intrinsic low-resolution prior calculated with ITP from the scattering curve for $s \leq 2.88 \mathrm{~nm}^{-1}$ is chosen. If the high-resolution prior, which was derived from the crystal structure, is used, only a minor shift (about $0.02 \mathrm{~nm}$ ) is visible. This demonstrates that the shifts

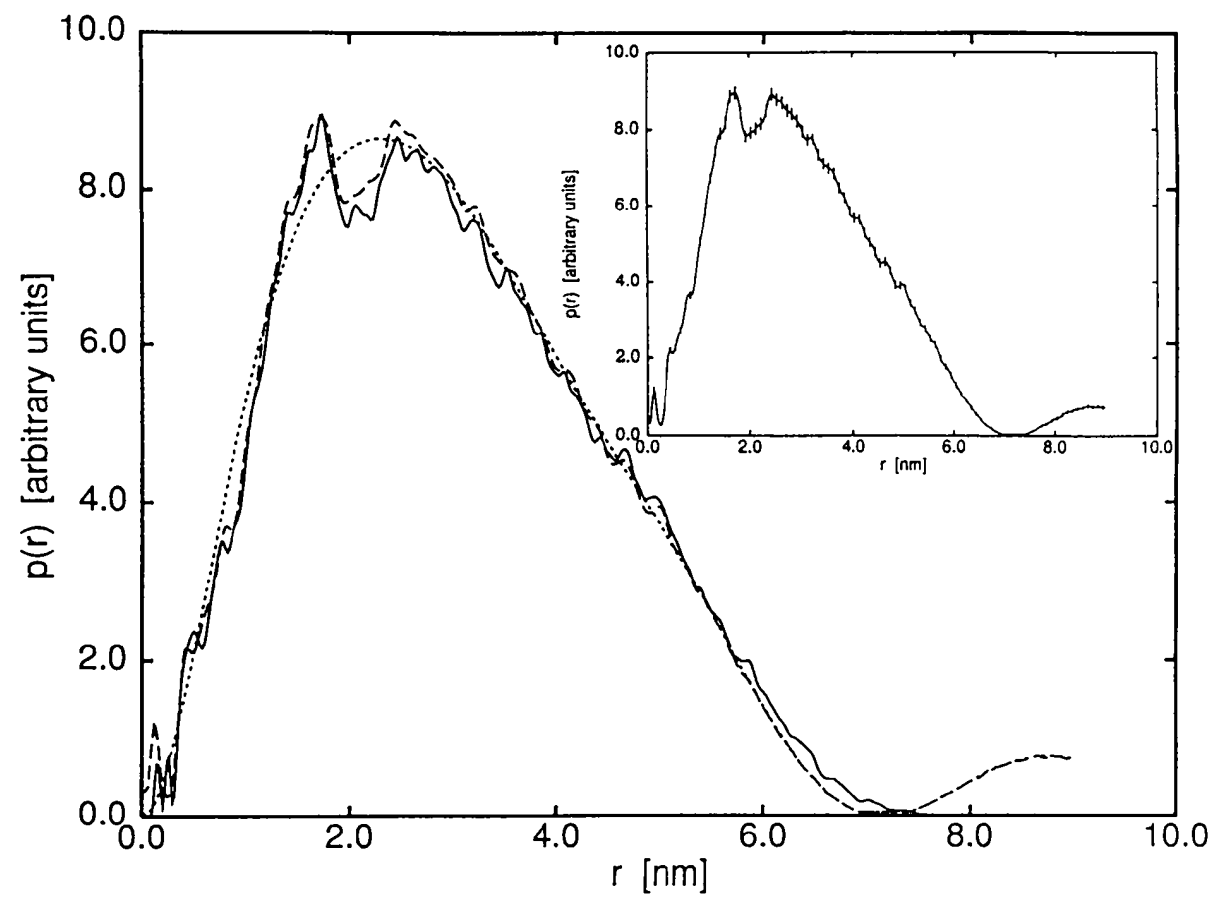

Fig. 20. MAXENT result for a modified tRNA structure using the low-resolution intrinsic prior. Full line: $p(r)$ of the modified tRNA structure. Short dashes: low-resolution intrinsic prior, calculated by direct Fourier transformation. Long dashes: $M A X E N T$ result. Insert shows error bars on the MAXENT result. 
are determined by the prior and cannot be used for any conclusions about structural changes.

All fine-structure features in the $p(r)$ of the crystal structure are visible in the $M A X E N T$ result for the experimental scattering curve. The $M A X E N T$ results with the intrinsic and the high-resolution model priors agree very well globally, giving the model a high degree of credibility. If we use the modified tRNA structure (which has been discussed above) as a high-resolution model prior, significant large-scale differences are visible between the results of $M A X E N T$ for the intrinsic prior and this model prior (not shown). By this comparison, it can be concluded

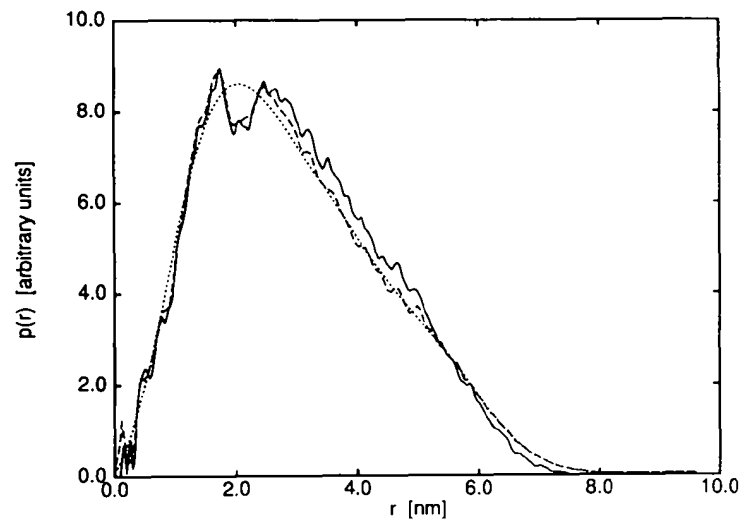

Fig. 21. MAXENT result for a modified tRNA structure using the low-resolution distance distribution of tRNA with crystal structure (4TRA) as model prior. Full line: $p(r)$ of the modified tRNA structure. Short dashes: low-resolution model prior, calculated by ITP (Glatter, 1977). Long dashes: $M A X E N T$ result. that the strongly modified crystal structure is an inappropriate model for the molecule in solution.

The entropy values for the different models are given in Table 2 . Again the values confirm the findings above.

From the discussion above, we conclude that the resulting structure of the tRNA in solution is identical with the crystal structure at $\pi / 15.5 \mathrm{~nm}$ resolution and no conformational changes can be detected for $t R N A$ during the transition from crystal to solution. The reason for the significant discrepancies between experimental and theoretical scattering curves for $s>3 \mathrm{~nm}^{-1}$ (Fig. 23) is unclear. The main differences

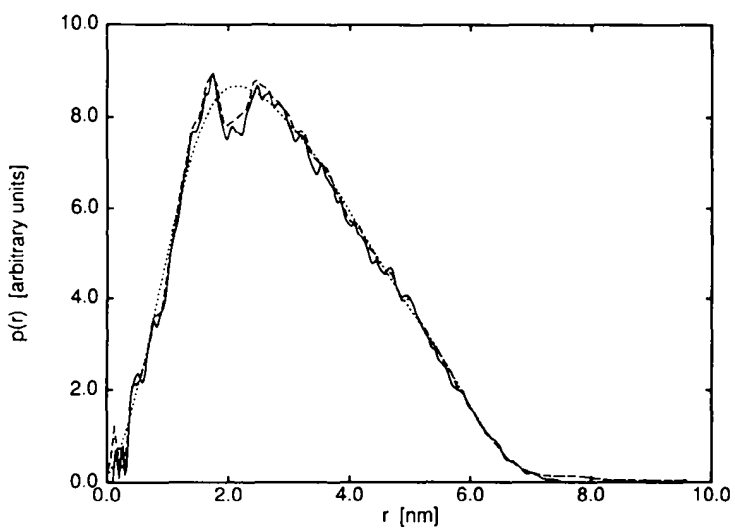

Fig. 22. MAXENT result for a modified tRNA structure using the low-resolution intrinsic prior. Full line: $p(r)$ of the modified tRNA structure. Short dashes: low-resolution intrinsic prior, calculated by ITP. Long dashes: MAXENT result.

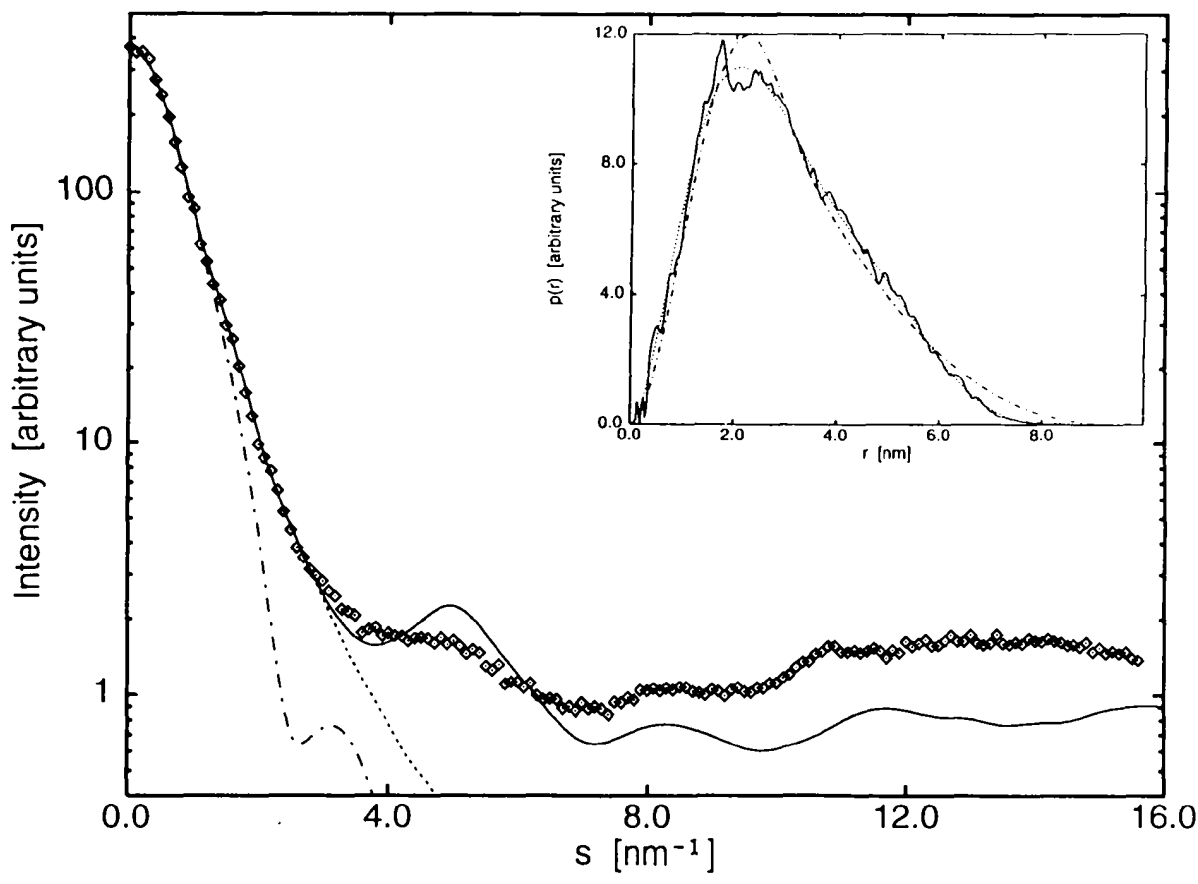

Fig. 23. Experimental scattering curve of RNA $^{\text {Phe }}$ in comparison with the scattering curve of tRNA in crystal (4TRA) and the scattering curves of priors. Markers show the experimental data. Full line: scattering curve of tRNA with crystal structure (4TRA). Dashed-dotted line: scattering curve of the rotational ellipsoid used as model prior $(A=4.95, \quad B=C=1.65 \mathrm{~nm})$. Short dashes: low-resolution scattering curve used for the intrinsic low-resolution prior. Insert: priors used for experimental tRNA $^{\text {Phe }}$ data analysis by $M A X$ $E N T$. Full line in insert: $p(r)$ of tRNA with crystal structure (4TRA). Dashed-dotted line in insert: $p(r)$ of rotational ellipsoid $(A=4.95, B=C=1.65 \mathrm{~nm})$. Short dashes in insert: intrinsic lowresolution prior. 
Table 1. Entropy c'alculated by MAXENT for different priors for the modified $t R N A$ structure

$\begin{array}{lccc}\text { Prior } & \begin{array}{c}\text { Method of } \\ \text { calculation }\end{array} & \text { Figure } & \text { Entropy } \\ \begin{array}{l}\text { Model, high resolution 4TRA } \\ \text { Model, medium resolution }\end{array} & \text { Direct } & 18 & -1.08 \\ \quad \text { 4TRA } & \text { Direct } & 19 & -1.32 \\ \begin{array}{l}\text { Model, rotational ellipsoid } \\ \text { 4TRA }\end{array} & \text { Direct } & - & -4.24 \\ \begin{array}{l}\text { Intrinsic, low resolution } \\ \text { Intrinsic, medium resolution }\end{array} & \begin{array}{c}\text { Direct } \\ \text { Model, medium resolution }\end{array} & 20 & -2.00 \\ \quad \text { DTRA } & \text { ITP } & & -1.26 \\ \quad \begin{array}{l}\text { Model, low resolution, 4TRA } \\ \text { Intrinsic, low resolution 4TRA }\end{array} & \text { ITP } & 21 & -1.04 \\ \quad \text { Intrinsic, medium resolution } & \text { ITP } & 22 & -1.13 \\ \quad \text { 4TRA } & \text { ITP } & & -1.02\end{array}$

between the $M A X E N T$ result for the experimental tRNA $^{\text {Phe }}$ and the $p(r)$ function calculated from the crystal structure are localized in the region between the 1.73 and $2.5 \mathrm{~nm}$ maxima. The minimum is filled up by electron-density inhomogeneities with distances close to the cross section of the RNA double helices, which is somewhat larger than the phosphate-phosphate distances across the arms of tRNA. The scattering contributions of counterions near the negatively charged phosphates are not included in the theoretical calculations, which may explain the differences between the scattering curves and distancedistribution functions.

The subtraction of an incorrect background may shift the fine structure or cause a splitting of the maxima but this effect will be discussed elsewhere.
Table 2. Entropy calculated by MAXENT for different priors for the experimental $t R N A^{\text {Phe }}$ data

Prior

$\begin{array}{cc}\begin{array}{c}\text { Method of } \\ \text { calculation }\end{array} & \text { Entropy } \\ & \\ \text { Direct } & -5.93 \\ \text { Direct } & -3.27 \\ \text { Direct } & -3.62 \\ I T P & -3.60\end{array}$

\section{Concluding remarks}

$M A X E N T$ represents a method that can be used for the analysis of small- and wide-angle X-ray and neutron scattering data. It combines the advantages of direct and conventional indirect methods. High resolution for larger molecules is available, no explicit scattering-curve extrapolation is necessary and noise of realistic levels can be smoothed for measurements at point distances of $1 / 2$ to $1 / 6$ sampling-point distance. $M A X E N T$ also makes it possible to use $a$ priori knowledge about the scattering system but this is not mandatory. The concept of intrinsic priors and model priors allows the user to apply $M A X E N T$ in a classical sense without any a priori knowledge or to introduce models and prove their consistency with the experimental scattering data. The best results with intrinsic priors were achieved by a two-step procedure. The first step is the calculation of a low-resolution prior from the experimental scattering data (e.g. by the ITP program). In the next step, the resolution is enhanced by $M A X E N T$ up to the maximum available value. To test models, a

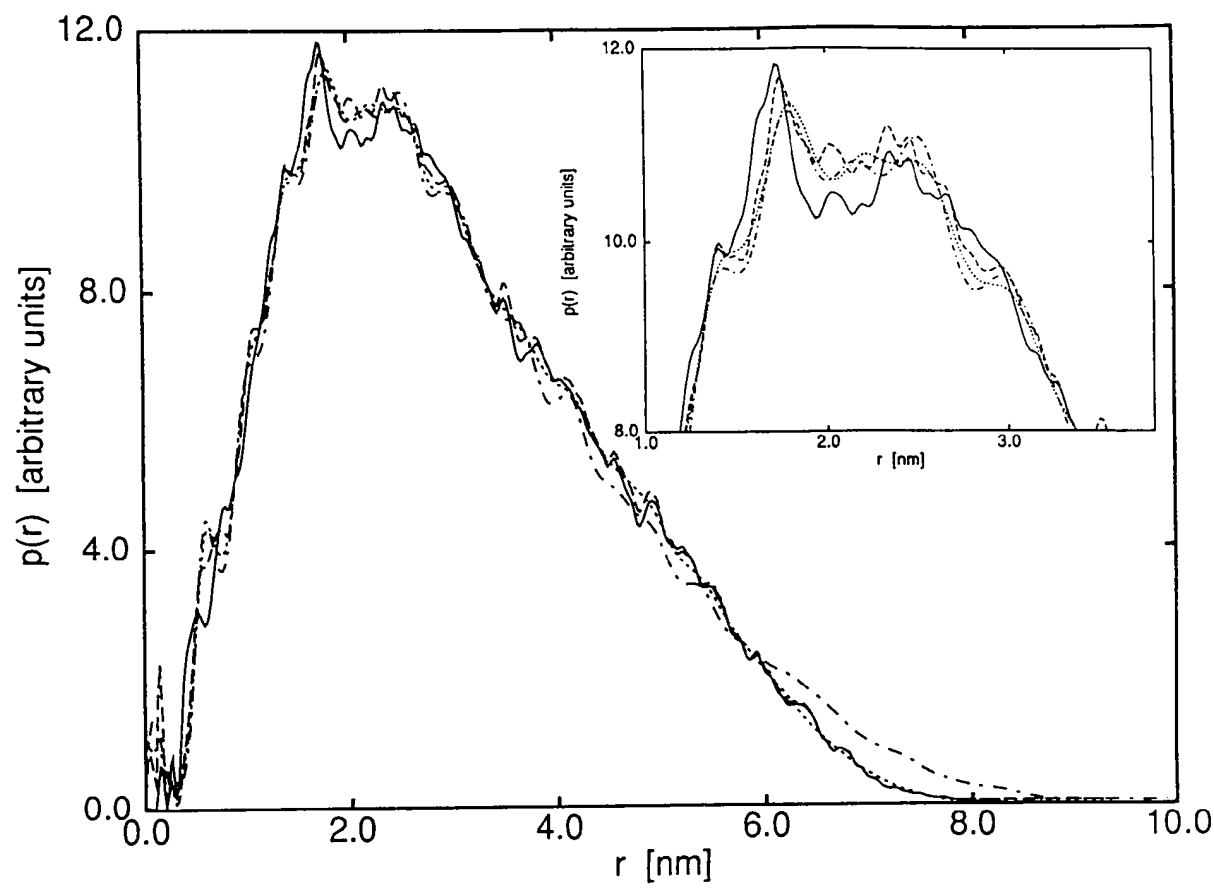

Fig. 24. MAXENT results for experimental tRNA ${ }^{\text {Phe }}$ using intrinsic and model priors. Full line: $p(r)$ of IRNA with crystal structure (4TRA). Short dashes: $M A X E N T$ result using intrinsic low-resolution prior. Dasheddotted line: MAXENT result using ellipsoidal model prior. Long dashes: $M A X E N T$ result using high-resolution model prior 4TRA. Insert: maximum region of $p(r)$. 
comparative study of $M A X E N T$ results for intrinsic and model priors is recommended. The best of the models can be selected by comparison of the entropy values. The resolution in $p(r)$ is not restricted by $M A X E N T$, neither is the largest dimension of the scatterer.

The program $M A X E N T$ is available from $\mathrm{SH}$. It is written in Fortran 77, uses no package routines and is as such easily portable to any computer. A version written in $\mathrm{C}$ is also available.

This work was supported by grants from the Deutsche Forschungsgemeinschaft (Mu 989/1-1), from the Bundesministerium für Forschung und Technologie (0310188AO) and from the Danish Natural Science Research Council.

\section{References}

Bernstein, F. C., Koetzle, T. F., Williams, G. J. B., Meyer, E. F., Brice, M. D., Rodgers, J. R., Kennard, O., Swimanouchi, T. \& Tasumi, M. (1977). J. Mol. Biol. 112 , 535-542.

BRYAN, R. K. (1990). Eur. Biophys. J. 18, 165-174.

Daniell, G. J., Potton, J. A. \& Rainford, B. D. (1988a). J. Appl. Cryst. 21, 891-897

Daniell, G. J., Potton, J. A. \& Rainford, B. D. (1988b). J. Appl. Cryst. 21, 663-668.

Glatter, O. (1977). J. Appl. Cryst. 10, 415-421.

Glatter, O. (1982). Small Angle X-ray Scattering, edited by O. Glatter and O. Kratky. London: Academic Press.

Guinier, A. \& Fournet, G. (1955). Small-Angle Scattering of $X$-rays. New York: Wiley.
Gull, S. F. (1989). Maximum-Entropy and Bayesian Methods, edited by J. Skilling, pp. 53-71. Dordrecht: Kluwer Academic Publishers.

Hansen, S. \& Pedersen, J. S. (1991). J. Appl. Cryst. 24, 541-548.

Harvey, S. C. \& MCCammon, J. A. (1981). Nature (London), 294, 286-287.

JAYNeS, E. T. (1983). Papers on Probability, Statistics and Statistical Physics, edited by R. D. Rosenkrantz. Dordrecht: Kluwer Academic Publishers.

KullbaCK, S. (1959). Information Theory and Statistics, pp. 142 and 190. New York: Wiley.

Marquardt, D. W. (1963). J. Soc. Ind. Appl. Math. 2, 431-469.

Morrison, J. D., Corcoran, J. D. \& Lewis, K. E. (1992) J. Appl. Cryst. 25, 504-513.

Múleer, J. J. (1983). J. Appl. Cryst. 16, 74-82.

MÚller, J. J. (1992). Program Package APX63, Small-Angle, Part 4, Data Evaluation, pp. 1-147. Freiberg: Freiberger Präzisionsmechanik $\mathrm{GmbH}$,

Múller, J. J., Damaschun, G. \& Schrauber, H. (1990). J. Appl. Cryst. 23, 26-34.

Múller, J. J., Damaschun, G., Wilhelm, P., Welfle, H. \& Pilz, I. (1982). Int. J. Biol. Macromol. 4, 289-296.

Múller, J. J. \& Schrauber, H. (1992). J. Appl. Cryst. 25, 181-191.

SkILling, J. (1988). Maximum-Entropy and Bayesian Methods in Science and Engineering; Vol. 1, edited by G. J. Erickson and C. Ray Smith, pp. 173-187. Dordrecht: Kluwer Academic Publishers.

SkILling, J. (1989). Maximum-Entropy and Bayesian Methods, edited by J. Skiluing, pp. 42-52. Dordrecht: Kluwer Academic Publishers.

Steenstrup, S. (1985). Aust. J. Phys. 38, 319-327.

Tanaka, N., Yamane, T., Tsukihara, T., Ashida, T. \& KaKUDO, M. (1975). J. Biochem. (Tokyo) 77, 147-162.

Westhof, E., Dumas, P. \& Moras, D. (1988). Acta Cryst. A44, 112-123. 